\title{
Deep Venous Thrombosis Associated With Inferior Vena Cava Abnormalities And Hypoplastic Kidney In Siblings
}

\author{
Duicu Carmen*, Bucur Gabriela, Simu lunius, Tripon Florin, Marginean Oana
}

University of Medicine and Pharmacy of Tirgu Mures, Romania

Congenital inferior vena cava anomalies have a reduced frequency in general population, many times being an asymptomatic finding. Patients caring such anomalies are at risk to develop deep vein thrombosis. In this paper, we present 2 siblings with deep venous thrombosis and inferior vena cava abnormalities, with a symptomatic onset at similar age. The inferior vena cava abnormality was documented by an angioCT in each case. The thrombophilic workup was negative. Patients were treated with conservative therapy: low molecular weight heparin anticoagulants converted later to oral anticoagulant with resolution of symptoms and disappearance of the thrombus. Finally, in the absence of any risk factor in a young patient admitted with deep vein thrombosis investigations to exclude inferior vena cava anomalies are mandatory.

Keywords: inferior vena cava, thrombosis, agenesia, kidney hypoplasia

Received: 31 December 2015 / Accepted: 21 January 2016

\section{Introduction}

Agenesia of the inferior vena cava (IVC) is an uncommon vascular abnormality in children usually remaining asymptomatic in childhood [1]. It represents a well-known cause of ileo-femoral deep vein thrombosis (DVT) $[2,3]$. The incidence of IVC abnormalities in general population is $0.2-1 \%$; this incidence being higher in young adults with DVT, namely 5\% [2,4]. A precise diagnosis needs an angio-computed tomography (angio-CT) or angio-MRI (Magnetic resonance imaging) evaluation [4,5]. In the last years, several cases of DVT associated with IVC agenesia have been reported in children and adolescents, and these venous aberrancies have been proposed as a possible independent predisposing factor for thrombosis, especially in the young $[1,5]$. We present the case of a brother and sister with an episode of venous thrombosis at similar age being subsequently diagnosed with an anomaly of IVC.

\section{Case 1}

A 12-years old boy, with no significant past medical history, was admitted to the Pediatric Surgery Department with signs of acute abdomen. The patient underwent appendectomy with removal of a catarrhal appendix, meantime during peritoneal cavity exploration a retroperitoneal tumor $15 \mathrm{~cm}$ in length was found so an angio-CT was done.

There was no history of risk factors for venous thrombosis. He denied smoking, taking alcohol or illegal drugs, recent trauma, immobilization or strenuous physical activity.

Angio-CT revealed the absence of IVC from common iliac veins connection to the confluence of hepatic veins. The patient had a short segment of IVC between the nor-

* Correspondence to: Carmen Duicu

E-mail: duicucarmen@yahoo.com mal sized hepatic veins and the right atrium. The venous drainage from lower limbs and pelvis was found to be supplied by collateral circulation that includes prominently dilated veins: hemiazygos, paraspinal and mesenteric. Moreover, the angio-CT documented thrombus within right renal vein and azygos vein (Figure 1). The right kidney was enlarged 130/46 mm with light contrast enhancement at the renal cortex.

The Doppler ultrasound showed the common iliac veins, external iliac veins and common femoral veins, great saphenous veins were compressible with vascular flow. No venous flow have been detected at the right renal vein. IVC was detectable at the confluence of the suprahepatic veins.

Conservative treatment consisted of subcutaneous low molecular weight heparin (LMWH) for 2 weeks followed by oral anticoagulants (warfarin) plus antiplatelets and compression hosiery with good evolution.

One year later a MRI exam was done: hypoplasic right kidney $52 \mathrm{~mm}$ length, with altered structure; left kidney $109 \mathrm{~mm}$ in length, normal structure. The abdominal and pelvic veins are similar with previous exams. IVC is seen just to the hepatic veins level; under this point a mini-

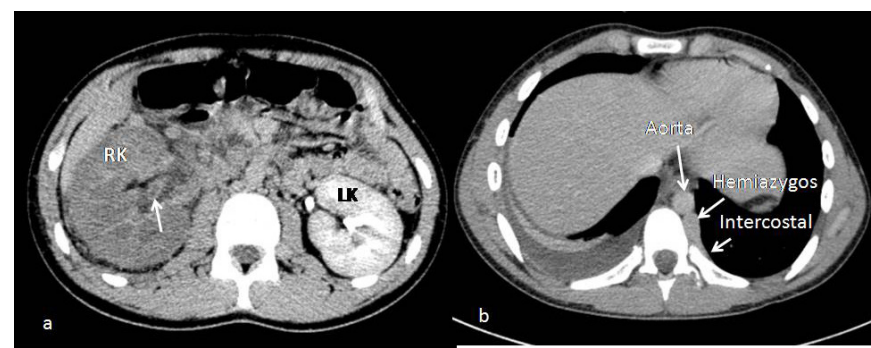

Fig. 1. Case 1- Contrast enhanced abdominal CT scan (axial): a. There is thrombus within the right renal vein; enlarged right kidney; b. Absence of IVC. White arrow from top to down: aorta, dilated hemyazigos vein, dilated intercostals vein 
mal collateral circulation is detected. The common iliac veins are not detected, while the external iliac veins have a normal diameter. In the retroperitoneum are seen few collateral veins, just in the left renal pelvis a richer venous plexus to the hemiazygos vein is discovered. The intervertebral and paravertebral veins are dilated. Portal, splenic and mesenteric veins have a normal caliber.

His follow-up revealed the disappearance of the right kidney. A DMSA renal scan demonstrated no right renal uptake and left renal uptake of approximately $99 \%$.

After $2 \frac{1}{2}$ years, the patient still takes oral anticoagulants, applies external elastic socking, and avoids thrombotic risk factors.

\section{Case 2}

A previously healthy 12-year-old girl, the sister of the previous case, presented to the Emergency Department with a history of 72-hours pain in right abdominal flank and followed by lower limb pain. Physical exam revealed normal vital signs and important swelling to the right thigh. A right lower limb venous duplex ultrasound demonstrated acute DVT within common femoral veins to the $3^{\text {rd }}$ distal external iliac vein.

Angio-CT exam revealed the absence of the supra and infrarenal segment of IVC, the venous circulation being balanced by collateral circulation through lumbalis and paravertebral veins with prominently dilated veins: azygos and hemiazygos. The angio-CT confirmed thrombus within right femoral vein to common and external iliac veins. The left kidney was hypoplastic with compensatory hypertrophied right kidney (Figure2). The adrenal glands have nodular calcifications.

The anticoagulant with LMWH and antiplatelets therapy was started, but because the right thigh pain and swelling of the lower limb extended to the tibial and fibular veins the full-dose of LMWH was initiated with significantly improved condition. Doppler ultrasound of the right lower limb demonstrated dilated common femoral veins with intraluminal echogenic mass which extend to posterior tibial veins, fibular veins; findings compatible with DVT.

She was discharged on this dose of LMWH for 1 month, than changed to long-term warfarin. The patient was advised to use fitted elastic stocking, and avoid thrombogenic

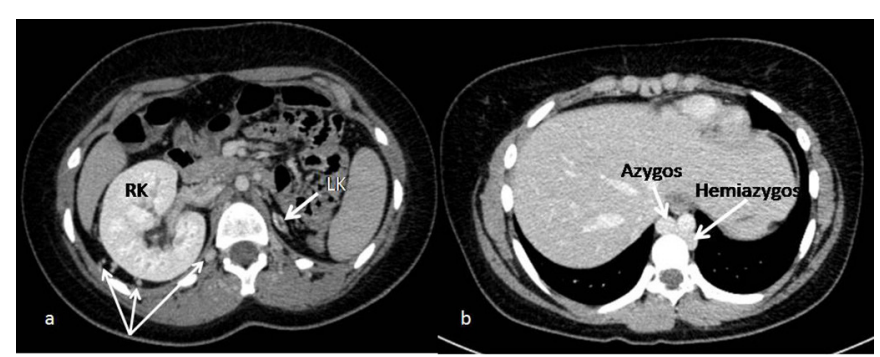

Fig. 2. Case 2- Angio-CT (axial): a. left kidney (LK) hypoplasia with compensatory right kidney(RK) hypertrophy; collaterals; b. azygos and hemiazugos vein, absence of IVC risk factors. At 1 and 3-month follow-up evaluations, her right lower limb was mild swelled with normal Doppler flow of the veins.

In order to have an accurate diagnosis and to rule out the differential diagnosis we completed investigation with additional lab tests; in both cases leucocytosis with neutrophilia; elevated CRP, D-dimmer and fibrinogen levels were found. Complete blood count, basic metabolic panel, INR, prothrombin time, activated partial thromboplastin time, uric acid, $\mathrm{LDH}$, electrolytes, liver function tests were all within the normal range.

No specific signs and symptoms related to a neoplasia, no lymph nodes enlargement, and hepatosplenomegaly have been observed in any case. Blood pressure was normal in both cases.

Both patients were screened for inherited and acquired defects in blood coagulation around 8 weeks after acute phase.

Antithrombin III, Protein C, Protein S, anti-factor II (prothrombin) antibodies, plasma homocysteine levels were in normal range (under LMWH treatment). Anticardiolipin IgM and IgG antibodies and antinuclear antibodies were not detected. Tests for rheumatoid factor, anti-cyclic citrullinated peptide antibodies were also negative. The antiphospholipid syndrome was ruled out in both cases.

Also we performed genetic analysis to determine mutations of factor V Leiden, prothrombin gene 20210A or methylene tetrahydrofolate reductase (MTHFR) C677T which consists of a $\mathrm{C}$ (cytosine) to $\mathrm{T}$ (thymine) transition at nucleotide 677 and leads to an alanine to valine substitution. No anomalies were detected.

They have another sister ( 9 years old) which we investigated but no anomalies were detected in this case.

\section{Discussion}

Embryogenesis of the IVC occurs during week 4-8 of gestation and represents a complex process. The normal IVC consists of four segments: hepatic, suprarenal, renal, and infrarenal. The most common anomalies of IVC are duplication of IVC, left sided IVC, IVC continued with intrathoracic azygos vein, IVC agenesis, retro aortic left renal vein [6].There are controversies in the literature about the etiology of IVC abnormalities. Some authors suggest that thrombosis on the IVC during intrauterine or the perinatal period with obliteration and subsequent resorbtion is the origin of its disappearance, hence no embryologic abnormalities are seen $[2,6-8]$.

The diagnosis on both cases was established with angioCT (the gold standard technique).

The association of IVC agenesia with other congenital anomalies, like kidney hypoplasia have already been described [2].

In the study performed by Sagban et al anomaly for the right kidney was found in 6\% of IVC agenesis cases while a hypoplastic left kidney was found in $2.7 \%$ respectively [9]. The atrophy of the previously normal kidney in our $1^{\text {st }}$ 
case can be due to longstanding poor perfusion or recurrent silent thrombosis secondary to vascular malformation. In the $2^{\text {nd }}$ case the renal hypoplasia was on left side, this being in contradiction with previous literature demonstrating right renal hypoplasia in similar situation based on the embryologic combinations described by Gayer et al [10]. The combination of DVT with IVC agenesia and kidney abnormalities is known as "KILT" syndrome (kidney anomaly, IVC anomaly, and leg thrombosis [11].

In case of IVC agenesia the collateral circulation develops before DVT but it is unable to assure the increased blood flow due to a major physical effort, thus producing venous stasis and clotting. There is a high risk of DVT recurrence because of inadequate venous return and consequent venous stasis. In case of lumbar DVT back pain is a common finding [3].

There is no standard treatment approach established for patients with DVT because of IVC agenesia. DVT events are rare in pediatric patients. There is no specific guideline about DVT treatment in pediatrics. LMWH is the preferred treatment in children.

Some authors recommend at least 6 months of anticoagulant treatment while in most cases life-long anticoagulation therapy is proposed even when thrombophilia investigation is negative in order to reduce the recurrence risk $[1,3,7,8]$. There are some reports of recurrence of thrombosis after discontinuation of the treatment [12]. The treatment was conducted according with clinical evolution and serial ultrasound aspects.

In situation of a young patient with DVT without any classical risk factor a complete investigation, including IVC imaging techniques, in order to rule out possible congenital venous anomalies is recommended [7]. Because of vascular abnormality anticoagulation therapy is necessary for life.

Therefore, patients with a diagnosed IVC anomaly should be advised against excessive exercise, prolonged immobilization, smoking and oral contraceptive use [8].

\section{Conclusion}

When a teenager presents with unexplained venous thrombosis an underlying IVC anomaly should be considered as a differential diagnosis. In those patients found to have an
IVC anomaly it is required to evaluate them for kidney abnormality.

To our knowledge this is the first report of a siblings affected by IVC and kidney abnormalities associated with thrombosis. In such family the imaging screening and genetic counseling is highly recommended because of high morbidity risk.

\section{Conflict of Interests}

The authors declare that there is no conflict of interests.

\section{Acknowledgement}

This research was supported by the Internal Research Grants of the University of Medicine and Pharmacy Tîrgu Mureş, Romania, contract no 29/11.12.2013.

\section{References}

1. Kalicki B, Sadecka M, Wawrzyniak A, et al. Absence of inferior vena cava in 14-year old boy associated with deep venous thrombosis and positive Mycoplasma pneumoniae serum antibodies- a case report. BMC Pediatr. 2015;15:40.

2. Bami S, Vazquez Y, Chorny V, Goldfisher R, Amodio J. Deep Venous Thrombosis of the Leg, Associated with Agenesis of the Infrarenal Inferior Vena Cava and Hypoplastic Left Kidney (KILT Syndrome) in a 14-Year-Old Child,". Case Reports Pediatr. 2015;2015:864047.

3. Lambert $M$, Marboeuf $P$, Midulla $M$, et al. Inferior vena cava agenesis and deep vein thrombosis: 10 patients and review of the literature. Vasc Med. 2010;15:451-459.

4. Pop Sorin, Opincaru lulianu. Anomalies of the inferior vena cava in patients with deep venous thrombosis. Pictorial essay. Medical Ultrasonography 2012;14:53-59.

5. Lesanu G, Balanescu R, Pacurar D, et al. Complex Malformation of the Inferior Vena Cava. Chirurgia 2014;109:259-262.

6. Spentzouris G, Zandian A, Cesmebasi A et al. The clinicalanatomy of the inferior vena cava: a review of common congenital anomalies and considerations for clinicians. Clinical Anatomy, 2014;27:1234-1243.

7. Konopka CL, Salame M, Padulla GA, Muradás RR, Batistella JC. Deep venous thrombosis associated with agenesis of IVC. J Vasc Bras 2010;9:196-169.

8. Sitwala PS, Ladia VM, Brahmbhatt PB, Jain V, Bajaj K. Inferior Vena Cava Anomaly: A Risk for Deep Vein Thrombosis N Am J Med Sci. 2014;6:601-603.

9. Sagban TA, Scharf RE, Wagenhäuser MU, et al. Elevated risk of thrombophilia in agenesis of the vena cava as a factor for deep vein thrombosis Sagban et al. Orphanet Journal of Rare Diseases 2015;10:3.

10. G. Gayer, R. Zissin, S. Strauss, M. Hertz. IVC anomalies and right renal aplasia detected on CT: a possible link? Abdominal Imaging. 2003;28:395-399.

11. Van Veen J, Hampton KK, Makris M. Kilt syndrome? $\mathrm{Br} J$ Haematol. 2002;118:1199-2000.

12. Gil RJ, Pérez AM, Arias JB, Pascual FB, Romero ES. Agenesis of the inferior vena cava associated with lower extremities and pelvic venous thrombosis. J Vasc Surg. 2006;44:1114-1116. 